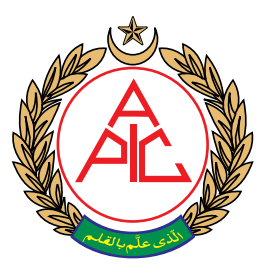

\title{
Does the time between ordering and administering the first dose of antibiotic influences outcome in septic shock patients?
}

\author{
Haifa M. Algethamy ${ }^{1}$, Abeer A. Arab ${ }^{2}$, Ayman Morish ${ }^{3}$, Lama H. \\ Meriky $^{4}$, Mohammad S. Numan ${ }^{5}$, Abdullah F. Alotaibi ${ }^{6}$
}

\footnotetext{
1,2,3 Department of Anesthesia and Critical Care Medicine, Faculty of Medicine, King Abdulaziz University Hospital, Jeddah, (Saudi Arabia)

${ }^{4}$ Resident, Department of Diagnostic Radiology, King Abdulaziz University Hospital, Jeddah, (Saudi Arabia)

${ }^{5}$ Department of Internal Medicine, King Fahad Armed Forces Hospital, Jeddah, (Saudi

Arabia); E-mail: Msaudnuman@ gmail.com.

${ }^{6}$ Medical student, Faculty of Medicine, King Abdulaziz University, Jeddah, (Saudi Arabia); E-mail: abdullmed17@gmail.com Correspondence:

Dr Haifa Mesfer Algethamy, Assistant Professor \& Consultant, Department of Anesthesia and Critical Care, King Abdulaziz University Hospital,

PO Box 80215, Jeddah, 21589,

(Saudi Arabia):

E-mail: Algethamy.Haifa@gmail. com

Received: 16 August 2019; Reviewed: 3, 5, 18 September 2019, Revised: 01 October 2019; Reviewed: 12, 21 October 2019; Accepted: 21 October 2019
}

\section{ABSTRACT}

Background \& Objectives: It is generally believed that significant delay in administering antibiotics in severely septic patients and those with septic shock increases mortality. However, most studies were retrospective and/or of questionable design. Moreover, the starting times from which delays were measured varied and often seemed somewhat amorphous. We assessed the duration of time between antibiotics being ordered and first administered among patients with newly diagnosed septic shock in a Saudi intensive care unit (ICU), and its effects on 30-day mortality and the rate of major complications. We also sought to identify any time threshold at which the mortality rate clearly increased.

Methodology: Data were prospectively collected on 96 patients $\geq 14$-years-old (male/ female $=49 \%$; mean age $62.1 \mathrm{y}$ ) admitted to our ICU and followed for $\geq 30$ days, or until hospital discharge or death. The time between ordering and administering the first dose of antibiotics after diagnosis of septic shock was recorded and its impact upon survival and major complications analyzed.

Results: Fifty of 96 patients died within the ICU. Unexpectedly, mortality rate declined steadily between < one min (60\%) and $5 \mathrm{~h}$ delay $(44 \%)$, but rose sharply beyond five hours $(p<0.001)$. Time delay did not significantly influence the rate of any major complication other than death.

Conclusions: Our results call into question recent conclusions that delays administering antibiotics beyond one to two hours result in significantly increased mortality. Further prospective, large scale studies are necessary to clarify this issue.

Key words: Sepsis; Septic shock; Antibiotics; Treatment; Mortality; Drug resistance

List of abbreviations: ICU: Intensive care unit BMI: Body mass index

SOFA: Sequential Organ Failure Assessment ESBL: Extended-spectrum betalactamase

MRSA: Methicillin-resistant Staph aureus

Citation: Algethamy HM, Arab AA, Morish A, Meriky LH, Numan MS, Alotaibi AF. Does the time between ordering and administering the first dose of antibiotic influence outcomes in septic shock patients? Anaesth pain \& intensive care 2019;23(3):340347, DOI: https://doi.org/10.35975/apic.v23i4.1165

\section{INTRODUCTION}

Every year, sepsis is diagnosed in up to three million
US residents and a minimum of 18 million people worldwide..$^{1-3}$ Overall mortality rates vary, but can reach $30-50 \%$, especially among those diagnosed with septic shock. ${ }^{3-5}$ 
Initiating antibiotics early is considered crucial to reducing mortality rates in sepsis patients, with or without septic shock, with many arguing that delays beyond one to two hours - after identifying sepsis and collecting appropriate culture samples increase mortality. ${ }^{4,9,10}$ This recommendation appears to stem largely from four published papers. In 2014, Ferrer et al. performed a retrospective analysis of extracted data, for the period of January 2005 through February 2010, from 165 intensive care units (ICUs) across Europe, the United States (US), and South America. ${ }^{4}$ Across the 165 centers, 28,150 patients met the criteria for either severe sepsis or septic shock, among which, 17,990 had received antibiotics after sepsis was identified. These investigators identified a statistically-significant increase in the probability of death associated with the number of hours of delay for first antibiotic administration, with in-hospital mortality rising steadily beyond a onehour delay, when adjusted for sepsis severity, route of admission to the ICU (via the emergency department vs. ward vs. another ICU), and geographic region, and independent of the number of organ systems in failure. Earlier, Kumar et al. published the results of their retrospective cohort study, performed from 1989 to 2004, of 14 ICUs and ten hospitals (4 academic, 6 community hospitals) in Canada and the US and, among 2,154 septic shock patients who received effective antimicrobial therapy only after the onset of recurrent or persistent hypotension, a strong relationship between the delay in effective antimicrobial initiation and in-hospital mortality was noted [adjusted odds ratio 1.12 (per hour delay), $\mathrm{p}<.0001$ ], with each hour of delay in antimicrobial administration beyond one hour decreasing survival by a mean $7.6 \%$ over the ensuing six hours. ${ }^{11}$ In this population, the median time to effective antimicrobial therapy was six hours.

In another retrospective study that incorporated 6 hospitals and 45 clinics within a single US healthcare system, for which data from January 2011 through July 2015 were extracted, Pruinelli et al. sought to determine the time-delay threshold for completion of each of the four Surviving Sepsis Campaign guidelines. ${ }^{10,12}$ These guidelines include; 1 ) obtaining pre-antibiotic blood culture; 2) obtaining a serum lactate level; 3) administering broad-spectrum antibiotics; and 4) administering $30 \mathrm{~mL} / \mathrm{kg}$ of crystalloid fluid for hypotension - after which mortality rates increased. Statistically significant threshold times for these four recommendations were: 20 min for obtaining a serum lactate sample; $50 \mathrm{~min}$ for obtaining blood cultures; $100 \mathrm{~min}$ for administering crystalloids; and $125 \mathrm{~min}$ for initiating broad-spectrum antibiotic therapy. The lack of randomized or prospective studies addressing the timing of antibiotic initiation was mentioned in their Cochrane Database Systematic review by Siddiqui et al., as a major concern limiting conclusions on the impact of antibiotic timing on survival. ${ }^{13}$

In the only prospective study of the four suggesting delay thresholds, Gaieski et al. assessed 261 patients with severe sepsis or septic shock admitted through the emergency department, from 2005 through 2006, and identified a significantly-reduced rate of mortality ( 19.5 vs. $33.2 \%$; odds ratio, $0.30 ; p=0.02$ ) among patients started on appropriate antibiotics within one hour of qualification for early goal-directed therapy versus afterwards. ${ }^{14}$ Despite the prospective nature of this study, it was small, indicating a need for subsequent prospective studies addressing the same issue.

The current study addresses this issue of insufficient prospective data on antibiotic initiation delays, focusing on a very practical issue: specifically addressing the duration of time between antibiotics first being ordered and first being administered. Specific goals were (1) to the range of delay between first antibiotic orders and administration among septic shock patients in the intensive care unit (ICU) of a major tertiary care center in Saudi Arabia; (2) to identify any impact of delay on outcomes: specifically, on ICU survival and the number of major complications (e.g., acute lung or kidney injury); and (3) to identify the time threshold at which further delays in antibiotic administration impact these outcomes.

\section{MOTHODOLOGY}

Prior to data collection, the study protocol was approved by the institution's ethics review board for research and is in full compliance with the second edition of the Declaration of Helsinki.

All patients were referred to the Intensive Care Unit (ICU) at King Abdulaziz University Hospital in Jeddah for treatment of septic shock over the fourteen months between 1 December 2015 and 31 January 2017. For the purposes of the current analysis, septic shock was defined as in the 'Third International Consensus Definitions for Sepsis and Septic Shock'. They defined septic shock as "a subtype of sepsis in which underlying circulatory and cellular/metabolic abnormalities are profound enough to substantially increase mortality". 5 Patients recruited early prior to publication of these consensus definitions, who did not meet the criteria for septic shock, were excluded from further analysis and will not be mentioned further in this paper.

The inclusion criteria included; diagnosis with septic shock (according to the latest definition on 2016), with age $\geq 14 \mathrm{y}$, did not have previous culture, not on antibiotics at the time of recruitment and ordered antibiotics within $24 \mathrm{~h}$ of the diagnosis of septic 
shock. Such patients were followed for 30 days, or till ICU discharge or death, whichever was sooner.

Patient eligibility for the study was determined by the study team at the time of their admission to the ICU, with all subsequent data either recorded electronically or using a pre-determined data-collection form.

Patients received standard care for septic shock and sepsis, which included the use of vasopressors if indicated; fluid resuscitation; supplemental oxygen; mechanical ventilation if indicated; and the empirical administration of antibiotics. The choice of all treatments was left to the treating team, in response to each patient's individual clinical picture. Standard monitoring included constant monitoring of vital signs, fluid intake and urine output, and regular monitoring of mental status. Standard laboratory investigations included at least daily blood samples to measure serum electrolytes, lactate, creatinine, liver function tests, cell counts, and any other lab tests or imaging deemed relevant to the individual case.

Antibiotic was chosen according to the international and national guidelines, depending upon the site of infection, hemodynamic instability, comorbidities and risk factors for the patient presenting with septic shock. All septic shock patients were assessed for their response to antibiotic administration according to their age, comorbidities, SOFA score, complications related to septic shock e.g. acute lung, liver or kidney injuries.

Data of specific interest included age, gender and nationality/race, height, weight, calculated body mass index (BMI), route of admission to the ICU, any comorbid conditions, the time and date when sepsis was diagnosed, the time and date when antibiotics were first ordered, the time and date when the first dose of antibiotic was administered, the anatomical site and established source of infection (e.g., respiratory tract, urinary tract, skin etc.), clinical tests, culture and sensitivity results and their source, other treatments administered, and various clinical outcomes, including various complications of sepsis - like acute lung and renal injury, need for dialysis or mechanical ventilation - a scientifically-validated general measure of clinical status (SOFA score, see below), and the patient's final disposal (e.g., death, continued hospitalization in the ICU or post-ICU ward, or discharge home).

As a measure of general clinical status, on ICU day \#1 (the day of admission) and ICU day \#3, each surviving patient's SOFA (Sepsis-related Organ Failure Assessment) score was calculated. ${ }^{15}$

\section{Statistical analysis:}

Prior to any data analysis, all data were screened for outliers and data errors, which were corrected. Continuous variables were summarized as means with ranges, while categorical variables were categorized as proportions. For inter-group comparisons involving two groups, continuous variables were compared by Student's t-tests when the data was normally distributed, and by Wilcoxen rank sums tests when not normally distributed. When three or more patient groups were compared, analysis of variance (ANOVA), with or without a conservative adjustment of degrees of freedom, was used, again depending on whether the data were parametric or non-parametric. Inter-group comparisons for all categorical variables, whether nominal or ordinal, were compared by Pearson $\chi 2$ analysis or Fisher's exact test, depending on the number of subjects per cell.

All tests were two-tailed, with $\mathrm{p} \leq 0.05$ set as the criterion for statistical significance, and $0.51<\mathrm{p} \leq$ 0.10 set as the criterion for borderline significance. All analyses were performed using the statistical software program SPSS, version 24.

\section{RESULTS}

Table 1: Demographic and baseline clinical characteristics of the sample

\begin{tabular}{l|c}
\hline Characteristic (N =96) & Mean or \% \\
\hline Age, mean (range) & $62.1(16-101)$ \\
\hline Age < 40 years & $13.5 \%$ \\
\hline Age 40-59 years & $27.1 \%$ \\
\hline Age 60-79 years & $43.8 \%$ \\
\hline Age $\geq 80$ years & $15.6 \%$ \\
\hline Male/Female & $48.9 \%$ \\
\hline Nationality - Saudi, $\mathrm{n}$ (\%) & $34.4 \%$ \\
\hline Nationality - Arab, $\mathrm{n}$ (\%) & $44.8 \%$ \\
\hline Nationality - Other, $\mathrm{n}$ (\%) & $19.8 \%$ \\
\hline Underweight, $\mathrm{n}$ (\%) & $6.3 \%$ \\
\hline Normal weight, $\mathrm{n}$ (\%) & $31.3 \%$ \\
\hline Overweight, $\mathrm{n}$ (\%) & $32.3 \%$ \\
\hline Obese, $\mathrm{n}$ (\%) & $29.2 \%$ \\
\hline At least one co-morbid condition at ICU admission & $93.8 \%$ \\
\hline Cardiovascular disease & $65.5 \%$ \\
\hline Pulmonary disease & $12.5 \%$ \\
\hline Kidney disease & $26.0 \%$ \\
\hline On dialysis & $13.5 \%$ \\
\hline Diabetes & $61.5 \%$ \\
\hline Cancer & $4.1 \%$ \\
\hline Immunosuppressed (AIDS or long-term steroids) & $2.1 \%$ \\
\hline Bedridden at ICU admission & $12.5 \%$ \\
\hline
\end{tabular}


Table 2: Culture results

\begin{tabular}{l|c} 
Organism grown & $\%$ \\
\hline Culture negative & $18.8 \%$ \\
\hline Gram positive bacteria identified & $16.7 \%$ \\
\hline Gram negative bacteria identified & $44.8 \%$ \\
\hline Gram positive + negative bacteria identified & $19.8 \%$ \\
\hline
\end{tabular}

Table 3: Culture / Sensitivity results

\begin{tabular}{l|c|c}
\multicolumn{1}{c|}{ Bacteria } & Percentage & $\begin{array}{c}\text { Percent } \\
\text { resistance }\end{array}$ \\
\hline E. coli & $27.1 \%$ & $69.2 \%$ \\
\hline Klebsiella & $22.9 \%$ & $22.7 \%$ \\
\hline Staph. Aureus & $15.6 \%$ & $40.0 \%$ \\
\hline Acinetobacter & $14.6 \%$ & $14.3 \%$ \\
\hline Enterococcus & $14.6 \%$ & $7.1 \%$ \\
\hline Strep. Pyogenes & $6.3 \%$ & $0.0 \%$ \\
\hline Stentotropomonas & $5.2 \%$ & $0.0 \%$ \\
\hline Strep. Pneumonia & $2.1 \%$ & $0.0 \%$ \\
\hline Providencia & $2.1 \%$ & $0.0 \%$ \\
\hline Other Staph. & $3.2 \%$ & $0.0 \%$ \\
\hline Other strep. & $2.1 \%$ & $0.0 \%$ \\
\hline Fungi & $5.2 \%$ & $\mathrm{n} / \mathrm{a}$ \\
\hline Influenza virus H1N1 & $1.0 \%$ & $\mathrm{n} / \mathrm{a}$ \\
\hline $\begin{array}{l}\text { Patients with } \geq \text { one } \\
\text { drug-resistant bacteria }\end{array}$ & $32.3 \%$ & $\mathrm{n} / \mathrm{a}$ \\
\hline $\begin{array}{l}\text { Percentage of culture }+ \\
\text { patients on appropriate } \\
\text { Abx }\end{array}$ & $60.4 \%$ & $\mathrm{n} / \mathrm{a}$ \\
\hline
\end{tabular}

\section{Characteristics of the overall sample}

A total of 96 patients met the selection criteria for the current analysis, for which the most pertinent demographic and baseline clinical data are summarized in Table 1.

Note that almost $60 \%$ of the sample $(59.4 \%)$ was age 60 or older, almost $80 \%$ was of Arab descent (79.2\%), and more than $60 \%(61.5 \%)$ were either overweight or obese. Almost all (94\%) had at least one co-morbid condition, the most common being cardiovascular disease, including hypertension (66\%), and diabetes mellitus (62\%). Immunosuppression was rare (just 2 of 96 patients) and cancer relatively uncommon (4\%). One patient in eight was bedridden prior to their admission to our ICU. In addition, 70\% of the patients were admitted to the ICU via the emergency room, while $28 \%$ were transferred from either a medical or a surgical service. The mean ICU day \# 1 SOFA score was 9.38, with scores ranging from 2 to 17.

Roughly, one of five patients (18.8\%) remained culture negative. Of the remainder, gram-negative cultures were almost three times as common as gram positive, with roughly $20 \%$ of patients growing at least one of each. The percentage of patients with each cultured organism is provided in Table 2.

Antibiotic resistance was very common amongst E. coli, with almost seven in ten E. coli cultures extended-spectrum beta-lactamase (ESBL) positive. Staph aureus was methicillin-resistant (MRSA) in $40 \%$ of cultures. The detailed results of culture / sensitivity tests are given in Table 3.

\section{Comparing time-delay thresholds}

Table 4 divides patients into two groups, based upon three different thresholds, in hours, indicating the delay between first antibiotics being ordered and initially administered. The following three twogroup comparisons are shown: (1) time-delay $\leq 1 \mathrm{~h}$ vs. $>1 \mathrm{~h}$; (2) time-delay $\leq 2 \mathrm{~h}$ vs. $>2 \mathrm{~h}$; and (3) time-delay $\leq 5 \mathrm{~h}$ vs. $>5 \mathrm{~h}$. Note that other timedelay thresholds (e.g., $3 \mathrm{~h}, 4 \mathrm{~h}$ and $6 \mathrm{~h}$ ) were analyzed, as well. However, these comparisons added no further insights to the three thresholds summarized in Table 4 , so they are not shown.

In terms of mortality within the ICU, only one comparison revealed a statistically-significant and clinically-significant result, that being the comparison of patients in whom the delay to antibiotic administration was $5 \mathrm{~h}$ or less vs. $5 \mathrm{~h}(\chi 2$ $=4.95$, df $1, \mathrm{p}=0.03)$. A clear rise in mortality rate is evident beyond five hours of delay in Figure 1. This rise remains graphically evident in Figure 2, where only patients who died in the ICU and patients discharged from the ICU were included in analysis, thereby adjusting for those remaining in either our ICU or another critical care unit beyond 30 days of follow-up, who might die in the ICU later. Note that only one in nine patients with a time-delay beyond $5 \mathrm{~h}$ survived and was discharged from the ICU over 30 days of follow-up, versus almost one in two of those with a time-delay of $\leq 5 \mathrm{~h}$. However, this difference failed to achieve even borderline statistical significance $(\mathrm{p}=0.13)$.

No inter-group comparison relating to the rate of four major complications - acute kidney injury, requirement for dialysis, acute lung injury, and required mechanical ventilation - yielded a significant result, though the rate of acute kidney injury was borderline higher in patients with a timeto-antibiotics time $>1 \mathrm{~h}$ versus their counterparts $\left(\chi^{2}=3.26, \mathrm{df} 1, \mathrm{p}=0.07\right)$. 
Table 4: Comparing outcomes in patients with different durations of delay starting antibiotics

\begin{tabular}{|c|c|c|c|}
\hline Outcome & $\leq 1.0$ hour & $>1.0$ hour & Statistical significance \\
\hline ICU death & $52.5 \%$ & $55.9 \%$ & $\chi^{2}=0.10 ; p=0.761$ \\
\hline Acute kidney injury & $56.7 \%$ & $75.0 \%$ & $\chi^{2}=3.26 ; p=0.073$ \\
\hline Dialysis required & $31.7 \%$ & $33.3 \%$ & $\chi 2=0.03 ; p=0.865$ \\
\hline Acute lung injury & $70.0 \%$ & $72.2 \%$ & $\chi 2=0.05 ; p=0.822$ \\
\hline Mechanical ventilation required & $58.3 \%$ & $66.7 \%$ & $\chi 2=0.66 ; p=0.417$ \\
\hline Outcome & $\begin{array}{l}\leq 2.0 \\
\text { hour2 }\end{array}$ & $\begin{array}{l}>2.0 \\
\text { hours }\end{array}$ & Statistical significance \\
\hline ICU death & $50.7 \%$ & $61.5 \%$ & $\chi^{2}=0.88 ; p=0.347$ \\
\hline Acute kidney injury & $58.8 \%$ & $75.0 \%$ & $\chi^{2}=2.24 ; p=0.132$ \\
\hline Dialysis required & $32.4 \%$ & $32.1 \%$ & $\chi^{2}<0.01 ; p=0.978$ \\
\hline Acute lung injury & $69.1 \%$ & $75.0 \%$ & $\chi^{2}=0.33 ; p=0.560$ \\
\hline Mechanical ventilation required & $58.8 \%$ & $67.9 \%$ & $\chi^{2}=0.68 ; p=0.413$ \\
\hline Outcome & $\begin{array}{l}\leq 5.0 \\
\text { hours }\end{array}$ & $\begin{array}{l}>5.0 \\
\text { hours }\end{array}$ & Statistical significance \\
\hline ICU death & $50.0 \%$ & $88.9 \%$ & $\chi^{2}=4.95 ; p=0.032$ \\
\hline Acute kidney injury & $63.5 \%$ & $63.6 \%$ & $\chi 2<0.01 ; p=0.985$ \\
\hline Dialysis required & $34.1 \%$ & $18.2 \%$ & $\chi 2=1.13 ; p=0.287$ \\
\hline Acute lung injury & $69.4 \%$ & $81.8 \%$ & $\chi^{2}=1.16 ; p=0.562$ \\
\hline Mechanical ventilation required & $60.0 \%$ & $72.7 \%$ & $\chi^{2}=0.67 ; p=0.411$ \\
\hline
\end{tabular}

\section{DISCUSSION}

We assessed a very specific and highly practical question, in response to our own hospital's decision, based on published guidelines, to have broadspectrum antibiotics immediately available in our ICU, as much as possible. The specific question was "Is there a time-threshold between ordering and administering antibiotics, which starts to impact patient survival, specifically in patients with septic shock?" This question differs in some ways and, we feel, is much more specific than the questions about time-to-antibiotic-administration that generally had been adopted for other studies, including those which Sherwin et al. analyzed together. ${ }^{9}$

One way in which our study differs from several of the others, relates to the decision we made based upon published Surviving Sepsis guidelines, to have broadspectrum antibiotics generally on-hand in the ICU for rapid administration. ${ }^{10}$ This decision led to 24 of our 96 patients having empirical antibiotics started within one minute of being ordered, 45 of 96 within $15 \mathrm{~min}, 60$ within one hour, and $68(71 \%)$ within 2 h. The median time-to-antibiotic administration across our 96 patients was $22 \mathrm{~min}$. This low median value contrasts considerably with the much longer administration on survival, the review authors claimed evidence supporting some impact of time in all but one, though the extent and conditions of that impact varied considerably. ${ }^{9,18}$ For example, in the study conducted by Jalilli et al., an impact of time only was apparent in the sickest patients with an APACHE score of 21 or higher. ${ }^{16}$ In the only randomized clinical trial, it was not the time duration to antibiotic administration, but whether or not the first dose of antibiotic was given before versus after the patient developed septic shock, which predicted survival. ${ }^{20}$ And in one of only three prospective cohort studies - two of which were considered methodologically poor and the other merely adequate - delay to antibiotic administration only affected survival beyond six hours. ${ }^{16,17,19}$

Our results were similar to this last, Norwegian study, in that we only identified an impact of time when the delay to antibiotic administration stretched beyond five hours. ${ }^{17}$ In fact, though not a statistically significant trend, there was a slow but steady decline in mortality rates from 'immediate' antibiotic administration (i.e., within one minute of the order being written; mortality rate $\sim 60 \%$ ) through to $5 \mathrm{~h}$ delay (mortality rate $44 \%$ ). One potential explanation for this could relate to a further difference between 
our study and the others cited by Sherwin, which is that we only analyzed patients in whom a diagnosis of septic shock had already been made, using current published guidelines. ${ }^{5}$ In such patients, as by Pruinelli et al. other steps might have greater priority in septic shock patients, like the administration of crystalloids to expand plasma volume and at least partially reverse severe hypotension, measuring serum lactate levels, and obtaining appropriate culture samples. ${ }^{12}$

In addition to identifying no increase in mortality until beyond $f \mathrm{~h}$ delay, we also identified no impact of time to antibiotics on the likelihood of acute lung or kidney injury, or on the need for dialysis or mechanical ventilation. To some extent, we must admit that such analysis is inherently difficult, given that some patients already were being dialyzed or were being mechanically ventilated prior to the recognition of septic shock.

\section{LIMITATIONS}

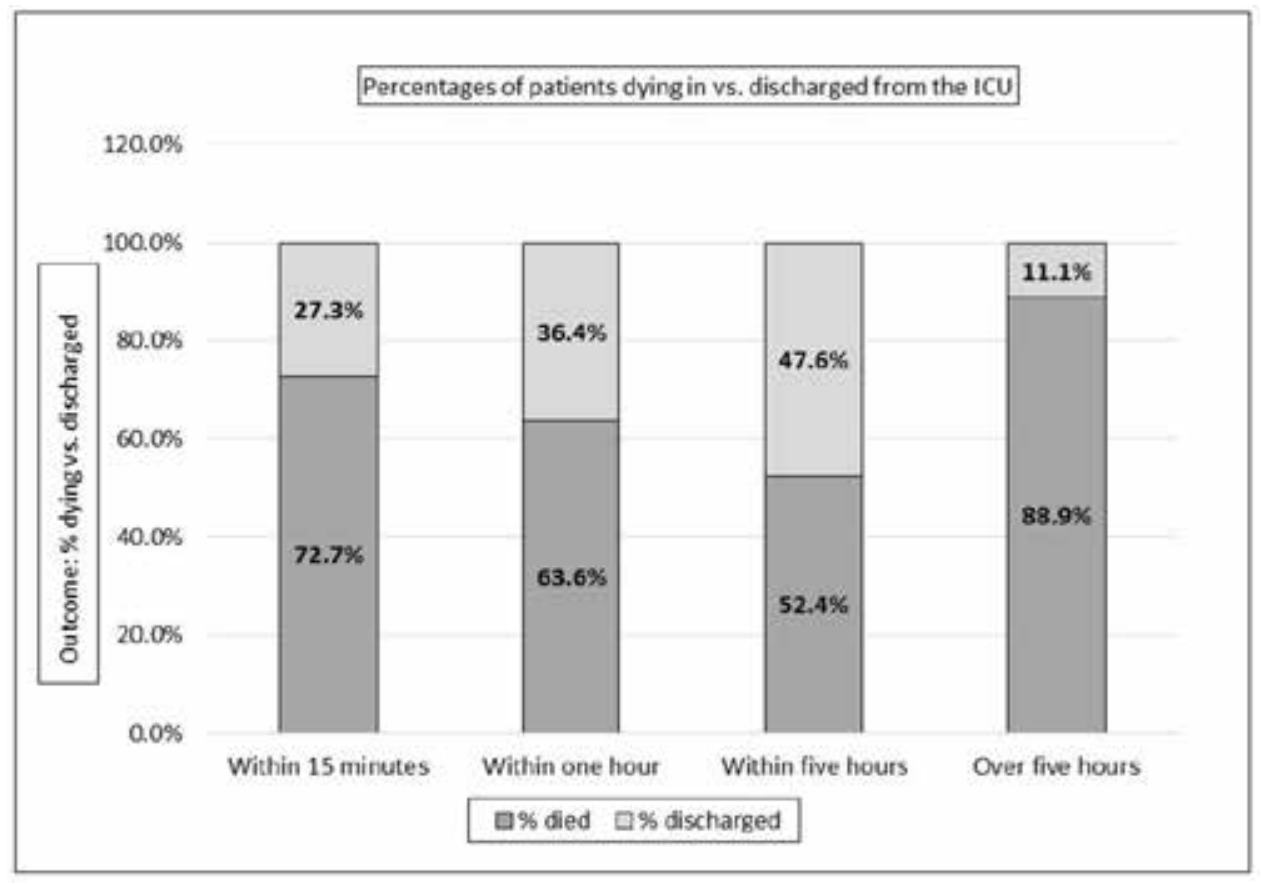

Figure 2: Percentage of patients dying in vs. discharged from the ICU.

This was a conscious decision, since of the 96 patients

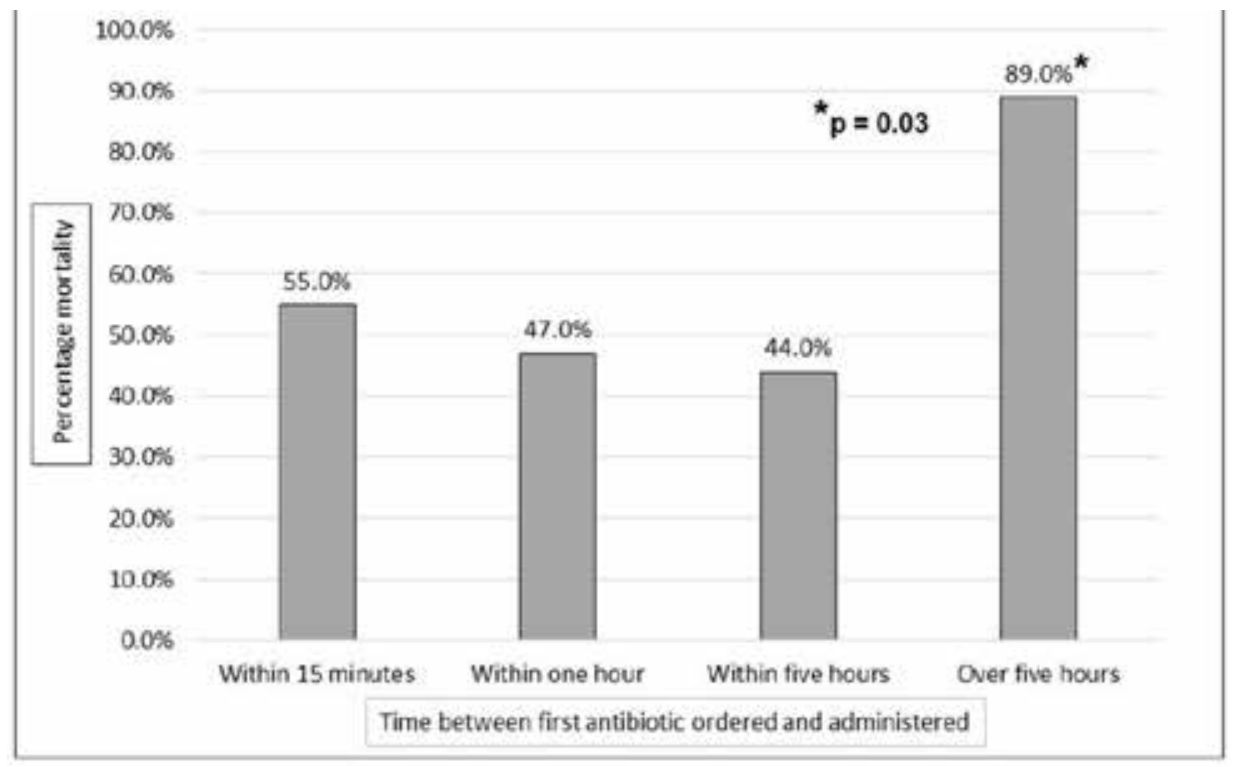

Figure 1: Percentage of ICU mortality by duration of delay between antibiotic order and administration

en tered into the
The most notable

limitation of the current study is clearly its relatively small size, in terms of patient numbers. On the other hand, it was prospective, and prospectively collected data is virtually always preferable, for a variety of reasons that include data completeness and $a$ priori adjustments for potential confounders.

We only looked at ICU and not in-hospital mortality. study, six remained as patients in our ICU, and 13 had been transferred to other units (e.g., surgical intensive care) and remained there beyond the 30 day observation limit of our study. To adjust for this, we repeated our analysis, only considering the fifty patients who died in the ICU and the 24 actively discharged to a lower level of care. Albeit not quite statistically significant, with this narrower sample, 
we once again noted the slow, gradual decline in mortality rates between immediate and 5-h antibiotic administration, followed by a sharp rise beyond five hours.

We also noted the exceedingly high rates of comorbidity in our sample, including roughly $65 \%$ with cardiovascular disease and over 60\% with diabetes, which is much higher than in some other studies; such co-morbidity could have biased our results. ${ }^{11}$

Our study was restricted to patients admitted to ICU, hence the sample size was small. We could have enhanced our sample size by involving other departments of the hospital or converted it into a multicenter study. This could have modified the results, hence we recommend larger studies to reach a definitive consensus.

\section{CONCLUSION}

Among 96 septic shock patients, ages 16 through 101, admitted to an ICU in Saudi Arabia, we were unable to detect any increase in mortality with delayed administration of broad-spectrum antibiotics until beyond five hours delay after the antibiotic was ordered, after which the rate of death rose dramatically. Further prospective studies are clearly needed, using clearly-demarcated and consistentlydocumented time points like the time of antibiotic orders, to clarify the impact of time to antibiotic administration on survival and other outcomes in septic shock patients.

Acknowledgements: We would like to thank Dr. Kevin White for his statistical support.

Ethics approval: The study was approved by the ethics committees at King Abdulaziz University Hospital vide Reference No. 431-17.

Confect of interests: All authors declare that they have no competing interests.

Funding: No funding was obtained for the study or preparation of this article

Authors' contribution:

HA - Primary author, critical revision and editing, generation of final manuscript

AA, LH, AM, MN and AA - Literature search, data collection, review of first version of manuscript. All authors have read and approved the final manuscript. 
original article

\section{REFERENCES}

1. Angus DC, Linde-Zwirble WT, Lidicker J, Clermont G, Carcillo J, Pinsky MR. Epidemiology of severe sepsis in the United States: analysis of incidence, outcome, and associated costs of care. Crit Care Med. 2001;29(7):1303-10. [PubMed] DOI: 10.1097/00003246200107000-00002

2. Gaieski D, Edwards J, Kallan M, Carr B. Benchmarking the incidence and mortality of severe sepsis in the United States. Crit Care Med. 2013;41(5):1167-74. [PubMed] DOl: 10.1097/CCM.0b013e31827c09f8

3. Slade E, Tamber PS, Vincent JL. The Surviving Sepsis Campaign: raising awareness to reduce mortality. Crit Care. 2003;7(1):1-2. [PubMed] DOI: 10.1186/cc1876

4. Ferrer R, Martin-Loeches I, Phillips G, Osborn TM, Townsend S, Dellinger $\mathrm{RP}$, et al. Empiric antibiotic treatment reduces mortality in severe sepsis and septic shock from the first hour: results from a guideline-based performance improvement program. Crit Care Med. 2014;42(8):1749-55. [PubMed] DOI: 10.1097/CCM.0000000000000330

5. Singer M, Deutschman CS, Seymour CW, Shankar-Hari M, Annane D, Bauer M. et al. The third international consensus definitions for sepsis and septic shock (Sepsis-3). JAMA. 2016;315(8):801-10. [PubMed] DOI: 10.1001/jama.2016.0287

6. Armstrong BA, Betzold RD, May AK. Sepsis and septic shock strategies. Surg Clin North Am. 2017;97(6):1339-79. [PubMed] DOl: 10.1016/i.suc.2017.07.003

7. Buckman SA, Turnbull IR, Mazuski JE. Empiric antibiotics for sepsis. Surg Infect (Larchmt). 2018;19(2):147-54. [PubMed] DOI: 10.1089/sur.2017.282

8. Septimus EJ, Coopersmith CM, Whittle J, Hale CP, Fishman NO, Kim TJ. Sepsis National Hospital Inpatient Quality Measure (SEP1). Multistakeholder work group recommendations for appropriate antibiotics for the treatment of sepsis. Clin Infect Dis. 2017;65(9):1565-9.
[PubMed] DOI: 10.1093/cid/cix603

9. Sherwin R, Winters ME, Vilke GM, Wardi G. Does early and appropriate antibiotic administration improve mortality in emergency department patients with severe sepsis or septic shock? J Emerg Med. 2017;53(4):588-95. [PubMed] DOl: 10.1016/i.jemermed.2016.12.009

10. Rhodes A, Evans LE, Alhazzani W, Levy MM, Antonelli M, Ferrer R, et al. Surviving sepsis campaign: international guidelines for management of sepsis and septic shock: 2016. Intensive Care Med. 2017;43(3):304-77. [PubMed] DOl: 10.1007/s00134-017-4683-6

11. Kumar A, Roberts D, Wood KE, Light B, Parrillo JE, Sharma S, et al. Duration of hypotension before initiation of effective antimicrobial therapy is the critical determinant of survival in human septic shock. Crit Care Med. 2006;34(6):158996. [PubMed] DOI: 10.1097/01. CCM.0000217961.75225.E9

12. Pruinelli L, Westra BL, Yadav P, Hoff A, Steinbach M, Kumar V, et al. Delay within the 3-hour surviving sepsis campaign guideline on mortality for patients with severe sepsis and septic shock. Crit Care Med. 2018 Apr;46(4):500-5. [PubMed] DOI: 10.1097/CCM.0000000000002949

13. Siddiqui S, Razzak J. Early versus late pre-intensive care unit admission broad spectrum antibiotics for severe sepsis in adults. Cochrane Database Syst Rev. 2010;(10):CD007081. [PubMed] DOI: 10.1002/14651858. CD007081.pub2

14. Gaieski DF, Mikkelsen ME, Band RA, Pines JM, Massone R, Furia FF, et al. Impact of time to antibiotics on survival in patients with severe sepsis or septic shock in whom early goaldirected therapy was initiated in the emergency department. Crit Care Med. 2010;38(4):1045-53. [PubMed] DOl: 10.1097/CCM.0b013e3181cc4824

15. Vincent JL, Moreno R, Takala J, Willatts S, De Mendonca A, Bruining H, et al. The SOFA (Sepsis-related Organ Failure Assessment) score to describe organ dysfunction/failure. On behalf of the Working Group on Sepsis-Related Problems of the European Society of Intensive Care Medicine. Intensive Care Med. 1996;22(7):707-10. [PubMed] D0I: 10.1007/bf01709751

16. Jalili $M$, Barzegari $H$, Pourtabatabaei N, Honarmand A, Boreiri M, Mehrvarz $A$, et al. Effect of door-to-antibiotic time on mortality of patients with sepsis in emergency department: a prospective cohort study. Acta Med Iran. 2013;51(7):454-60. [PubMed]

17. Nygård $S$, Langeland $\mathrm{N}$, Flaatten $H$, Fanebust $R$, Haugen 0 , Skrede S. Aetiology, antimicrobial therapy and outcome of patients with community acquired severe sepsis: a prospective study in a Norwegian university hospital. BMC Infect Dis. 2014 Mar;14:121. [PubMed] DOl: 10.1186/1471-2334-14-121

18. Vilella A, Seifert C. Timing and appropriateness of initial antibiotic therapy in newly presenting septic patients. Am J Emerg Med. 2014;32(1):7-13. [PubMed] DOI: 10.1016/i.ajem.2013.09.008

19. Siddiqui S, Salahuddin N, Raza A, Razzak J. How early do antibiotics have to be to impact mortality in severe sepsis? A prospective, observational study from an emergency department. $J$ Ayub Med Coll Abbottabad. 2009;21(4):106-10. [PubMed]

20. Puskarich MA, Trzeciak S, Shapiro NI, Arnold RC, Horton JM, Studnek $\mathrm{JR}$, et al. Association between timing of antibiotic administration and mortality from septic shock in patients treated with a quantitative resuscitation protocol. Crit Care Med. 2011;39(9):2066-70. [PubMed] DOI: 10.1097/CCM.0b013e31821e87ab

21. Parish B, Cooksley T, Haji-Michael P. Effectiveness of early antibiotic administration in septic patients with cancer. Acute Med. 2013;12(4):196200. [PubMed] 